
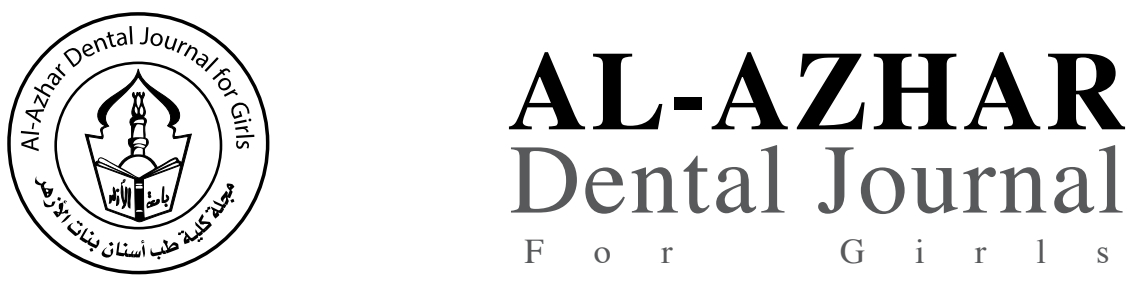

The Official Publication of The Faculty of Dental Medicine For Girls, Al-Azhar University Cairo, Egypt.

ADJ-for Grils, Vol. 4, No. 3, July (2017) — PP. 271:277

\title{
Evaluation of Lactate Dehydrogenase Activity During Orthodontic Tooth Movement
}

\author{
Amany M. Ibrahim ${ }^{(1)}$, Samir A. Ibrahim ${ }^{(2)}$, Tarek H. Marei ${ }^{(3)}$ and Hala H. Hazzaa ${ }^{(4)}$
}

Codex : 32/1707

dentaljournal.forgirls@yahoo.com

\begin{abstract}
Objective: This study was to evaluate the activity of Lactate Dehydrogenase enzyme in different tooth malpositions in gingival crevicular fluid (GCF). Patients, materials and methods: A sample of 18 patients with an age range from 13 to 19 years, requiring orthodontic treatment. The study groups were designed into two groups. Group I: well aligned teeth (control group). Group II: malposed teeth. Group II was subsequently divided into 3 sub-groups; Rotation, Spacing and Vertical malposition. Samples were collected for assessment of lactate dehydrogenase level in GCF at baseline, one week, two and four weeks after treatment. Lactate dehydrogenase was measured in GCF using Enzyme Linked Immunosorbent Assay (ELISA) technique. Results: In both groups (group I and group II subgroups) the gingival crevicular fluid lactate dehydrogenase enzyme had a steady increase during orthodontic tooth movement with a statistically significant increase at one week and two weeks compared with the base line $(\mathrm{P} \leq 0.05)$. No statistical significant difference was found between each two subgroups of group II $(\mathrm{P} \leq 0.05)$. Conclusions: The level of lactate dehydrogenase enzyme in gingival crevicular fluid could be used as a biomarker for periodontal metabolism related to orthodontic tooth movement. The level of lactate dehydrogenase enzyme (LDH) did not show significant difference among different tooth malpositions during correction by orthodontic treatment.
\end{abstract}

\section{INTRODUCTION}

Tooth movement by orthodontic treatment is characterized by remodeling changes in the periodontal ligament, alveolar bone, and gingiva. A reflection of this phenomenon can be found in the gingival
Lactate Dehydrogenase, GCF, ELISA.

1. Demonstrator, Department of Orthodontics, Faculty of Dental Medicine, Al-Azhar University for Girls.

2. Professor and Head of Orthodontic Department, Faculty of Dental Medicine, Al-Azhar University for Girls.

3. Assistant Professor, Department of Orthodontics, Faculty of Dental Medicine, Al-Azhar University for Girls.

4. Assistant Professor, Department of Oral medicine, Periodontology, Diagnosis and Radiology, Faculty of Dental Medicine, Al-Azhar University for Girls. 
crevicular fluid (GCF) of moving teeth, with significant elevations in the concentrations of its components ${ }^{(1-5)}$.

Mechanical forces often cause hyalinization leading to necrosis in the PDL and lead to bone resorption. Hyalinization occurs as cell-free areas of the PDL, in which the normal tissue architecture and staining characteristics of collagen in the processed histologic material have been lost. Distortions in the normal periodontal fiber arrangement were observed .Numerous cell fragments (debris), areas of degraded matrix interspersed between the intact collagen fibrils, and, in some cases, pyknotic nuclei were also present in hyalinization areas. In hypoxic conditions, cells will rely on anaerobic glycolysis. Many enzymes involved in an anaerobic metabolism can be potential markers ${ }^{(6-9)}$.

Paper extracted from Master thesis titled; (Evaluation of Lactate Dehydrogenase Activity during Orthodontic Tooth Movement.)

Lactate dehydrogenase is an example of a molecule that accumulates during anaerobic metabolism. Cells that adapt via metabolic changes will continue to live and cells that cannot adapt to the ischemic condition will die. The dead cell will lyse, releasing all of its contents to the milieu including Lactate dehydrogenase accumulated in cytoplasm ${ }^{(9-12)}$.

Lactate Dehydrogenase, an enzyme normally limited to the cytoplasm of cells, is only released extracellularly after cell death. Previous studies have demonstrated that the activity of lactate dehydrogenase in GCF is significantly correlated with gingival inflammation and tissue destruction from periodontitis ${ }^{(13-17)}$.

With the consideration that LDH is an index of tissue destruction, and that, during orthodontic tooth movement, phenomena such as cell necrosis, have been described in the periodontal ligament, an increase in LDH activity in the GCF can be hypothesized. The present study was carried out to evaluate the activity of Lactate Dehydrogenase enzyme in different tooth malpositions in gingival crevicular fluid.

\section{PATIENTS, MATERIALS AND METHODS.}

The study was performed on 18 female patients. The patients were selected from those who referred to the out-patient orthodontic clinic of Faculty of Dental Medicine, Al-Alzhar University for Girls.

\section{Criteria for patient selection:}

1. Age ranged from 13years to 19 years.

2. No history of previous orthodontic treatment.

3. No self-reported use of anti-inflammatory medications at previous 3 months.

4. Good periodontal health as evidenced by generalized probing depths $\leq 3 \mathrm{~mm}$ and no radiographic evidence of periodontal bone loss.

5. Full eruption of all permanent teeth with the exception of the third molars.

\section{The study groups:}

The samples were grouped according to alignment of teeth as following:

- Group I: Well aligned teeth (control group) (20 teeth).

- Group II: Malposed teeth: (study group) (45 teeth).

Group II was subsequently divided into 3 subgroups; Rotation, Spacing and Vertical malposition.

All patients put onto recall schedule at zero, one week, two and four weeks. Samples were collected for assessment of lactate dehydrogenase level in GCF and clinical parameters Probing depth, Gingival Index and Plaque Index at these time intervals mentioned.

At each interval, including the preliminary visit, repeated oral hygiene instructions relating to the correct use of a toothbrush, dental floss, an interdental brush and mouth wash were given to the subjects and inform them how to perform effective tooth cleaning in the presence of the orthodontic appliance. 


\section{Gingival crevicular fluid sampling}

Gingival Crevicular Fluid (GCF) samples were collected for evaluation of lactate dehydrogenase levels at baseline before initial orthodontic treatment, one week, two weeks, and four weeks after starting the treatment.

GCF samples were collected after the assessment of plaque index (PI) scores and before recording all clinical parameters. Moreover, no brushing procedures were allowed immediately before the GCF sampling to avoid mechanical injuries to the periodontal tissues.

Sample collections were done in the early morning. Each Crevicular site included in the study was isolated with cotton rolls. Before GCF collection, a gentle air stream was directed towards the tooth surface for 5 seconds to dry the area. GCF was collected in every site by using absorbable paper point (size 30) that was inserted into the gingival crevice until mild resistance was felt. The paper points were placed into the sulcus and waiting for $60 \mathrm{Sec}$, then transferred to the plastic Eppindrof (Microcentrifuge tubes, ISOLAB, Wertheim, Germany). Each sample stored at $-80^{\circ} \mathrm{C}$ until analysis. Contaminated paper points by blood or saliva were excluded from the study ${ }^{(18)}$.

\section{Analysis of Lactate dehydrogenase in GCF.}

Lactate dehydrogenase was measured in GCF samples by enzyme linked immunosorbent assay (ELISA) technique.

\section{RESULTS}

Lactate Dehydrogenase level: In both groups (group I and group II subgroups), the statistical analysis showed that the gingival crevicular fluid lactate dehydrogenase enzyme had a steady increase during orthodontic tooth movement with a statistically significant increase at one week and two weeks compared with the base line, while it showed decrease LDH level at four weeks in the two groups but still higher than base line $(\mathrm{P} \leq 0.05)$. Post hoc test revealed significant difference only between group I (well aligned) and each sub-group in group II (malposed) at two and four weeks of treatment. No significant difference was found between each two subgroups of group II (P>0.05). (Table 1)

Table (1) Concentration of Lactate dehydrogenase in both groups in different time intervals.

\begin{tabular}{|c|c|c|c|c|c|c|}
\hline \multicolumn{2}{|c|}{ Mean LDH (U/L) \pm SD } & at base line & After 1 wk of ttt & After 2 wks of ttt. & After 4 wks of ttt. & P value \\
\hline \multirow{2}{*}{ Groups } & Well aligned & $190.4 \pm 9.2$ & $206.1 \pm 10.6$ & $220.3 \pm 7.5$ & $210.6 \pm 6.9$ & $>0.001 *$ \\
\cline { 2 - 7 } & Rotated teeth & $193.4 \pm 13.8$ & $219.0 \pm 7.1$ & $275.9 \pm 25.2$ & $230.4 \pm 7.9$ & $>0.001 *$ \\
\hline \multirow{2}{*}{$\begin{array}{c}\text { Group II } \\
\text { subgroups }\end{array}$} & Spaced teeth & $194.7 \pm 9.6$ & $220.1 \pm 9.2$ & $264.1 \pm 25.5$ & $225.9 \pm 10.7$ & $>0.001 *$ \\
\cline { 2 - 7 } & Vertical malposed & $199.4 \pm 12.5$ & $216.6 \pm 8.1$ & $259.7 \pm 18.3$ & $222.1 \pm 14.5$ & $>0.001 *$ \\
\cline { 2 - 7 } & F value & 0.75 & 3.65 & 9.73 & 4.65 \\
\hline
\end{tabular}

$(P \leq 0.05)$. 
Probing depth: Although, There was no statistically significant difference between the two groups at all intervals, results showed significant difference in each group when a periods of treatment compared to base time $(\mathrm{P}>0.05)$. (Table 2$)$
Plaque Index (PI) and gingival index (GI): At both groups showed statistically insignificant difference between the two groups at base line, after one week, two weeks and four weeks intervals (P> 0.05). (Table 3 and 4)

Table (2) Probing depth in both groups in different time intervals and significance of difference using ANOVA test.

\begin{tabular}{|c|c|c|c|c|}
\hline \multirow{2}{*}{ Mean \pm SD } & Group I & \multicolumn{3}{|c|}{ Group II subgroup } \\
\cline { 2 - 5 } & Well aligned & Rotated teeth & Spaced teeth & Vertical malposed \\
\hline At base line & $30 \pm 0.87$ & $0.84 \pm 0.1$ & $0.79 \pm 0.3$ & $0.84 \pm 0.1$ \\
\hline After 1 wk. of ttt. & $30 \pm 1.03$ & $0.9 \pm 0.1$ & $1.06 \pm 0.4$ & $1.03 \pm 0.1$ \\
\hline After 2 wks of ttt. & $0.4 \pm 1.23$ & $1.30 \pm 0.4$ & $1.46 \pm 0.5$ & $1.30 \pm 0.4$ \\
\hline After 4 wks of ttt. & $1.41 \pm 0.4$ & $1.40 \pm 0.4$ & $1.36 \pm 0.4$ & $1.40 \pm 0.4$ \\
\hline F value & 7.44 & 4.98 & 3.15 & 4.54 \\
\hline \hline P value & $0.012^{*}$ & $0.008^{*}$ & $0.044^{*}$ & $0.012^{*}$ \\
\hline
\end{tabular}

$(P \leq 0.05)$.

Table (3) Gingival index in both groups in different time intervals and significance of difference using Kruskal Wallis Test.

\begin{tabular}{|c|c|c|c|c|}
\hline \multirow{2}{*}{ Mean \pm SD } & Group I & \multicolumn{2}{|c|}{ Group II subgroup } \\
\cline { 2 - 5 } & Well aligned & Rotated teeth & Spaced teeth & Vertical malposed \\
\hline At base line & $30 \pm 0.14$ & $0 \pm 0$ & $0.29 \pm 0.4$ & $0.14 \pm 0.3$ \\
\hline After 1 wk. of ttt. & $50 \pm 0.43$ & $0.14 \pm 0.3$ & $0.43 \pm 0.5$ & $0.43 \pm 0.3$ \\
\hline After 2 wks of ttt. & $0.5 \pm 0.57$ & $0.43 \pm 05$ & $0.29 \pm 0.4$ & $0.43 \pm 0.5$ \\
\hline After 4 wks of ttt. & $0.43 \pm 0.5$ & $0.43 \pm 0.5$ & $0.43 \pm 0.5$ & 2.70 \\
\hline Kruskal-Wallis value & 2.74 & 4.96 & 0.60 & 0.440 \\
\hline P value & 0.433 & 0.175 & 0.896 & \\
\hline
\end{tabular}

$(P \leq 0.05)$. 
Table (4) Plaque index in both groups in different time intervals and significance of difference using Kruskal Wallis Test.

\begin{tabular}{|c|c|c|c|c|}
\hline \multirow{2}{*}{ Mean \pm SD } & Group I & \multicolumn{3}{|c|}{ Group II subgroup } \\
\cline { 2 - 5 } & Well aligned & Rotated teeth & Spaced teeth & Vertical malposed \\
\hline At base line & $0 \pm 0$ & $0.14 \pm 0.3$ & $0.43 \pm 0.5$ & $0.14 \pm 0.3$ \\
\hline After 1 wk. of ttt. & $0 \pm 0$ & $0.43 \pm 0.5$ & $0.29 \pm 0.4$ & $0.43 \pm 0.5$ \\
\hline After 2 wks of ttt. & $0.29 \pm 0.4$ & $0.29 \pm 0.4$ & $0.29 \pm 0.4$ & $0.29 \pm 0.4$ \\
\hline After 4 wks of ttt. & $0.29 \pm 0.4$ & $0.43 \pm 0.5$ & $0.29 \pm 0.4$ & $0.43 \pm 0.5$ \\
\hline Kruskal-Wallis value & 4.50 & 1.74 & 0.47 & 1.74 \\
\hline P value & 0.212 & 0.629 & 0.925 & 0.629 \\
\hline
\end{tabular}

$(P \leq 0.05)$.

\section{DISCUSSION}

Orthodontic force could cause sequential reactions involving the periodontal tissue and alveolar bone, resulting in the release of numerous substances from the dental tissues and surrounding structures. For their importance in understanding the biological processes involved in orthodontic treatment, improve treatment, and reduce adverse side effects, these substances have been mentioned as biomarkers ${ }^{(5)}$. Moreover, it has been shown that hyalinization can occur in the most compressed areas of the periodontal ligament after application of orthodontic force on teeth. This hyaline zone has also been described as an area of focal aseptic necrosis ${ }^{(7)}$.

Lactate dehydrogenase is an oxido-reductase enzyme that is present within the cytoplasm of the cell and is released from cells during necrosis. During anaerobic condition, cells depend on anaerobic glycolysis for respiration, during which the end product, lactic acid, is formed. Lactate dehydrogenase helps in conversion of pyruvic acid into lactic $\operatorname{acid}^{(15)}$.

The results of this study showed that there were increased LDH levels during orthodontic tooth movement compared to the base line (before treatment) in all groups /sub groups and the mean of
LDH after two weeks of treatment was the highest mean. The results correlates with an earlier study which found that the levels of LDH increased in gingival crevicular fluid at the site where orthodontic force was applied compared to the base time ${ }^{(16)}$.

The LDH levels in all groups showed a steady increase from baseline, one week and two weeks. This was followed by a mild decrease from two to four weeks. The steep increase between one week and two weeks can probably be explained by Burrstone ${ }^{(19)}$ who found that there were three phases of orthodontic tooth movement- initial, lag and post-lag phases. Initial phase is characterized by movement of teeth within the periodontal ligament space. Lag phase is characterized by hyalinized zone where no tooth movement takes place.

Current study showed that there is no difference between different tooth malposition in level of LDH after exposed to initial orthodontic force, while there is significant difference when compared with well aligned teeth. Although, there is no significant difference in LDH level between malposed teeth sub groups (rotated, spaced and vertically malposed), the level of LDH showed slight elevation in rotated sub-group than the others after two weeks of treatment. This may be explained by a previous 
study ${ }^{(20)}$ which stated that diffuse distribution of forces applied to periodontal tissues during tooth movement tended not to promote extensive areas of cell matrix hyalinization. However, focal distribution or concentration of forces within a restricted area as it occurs in tipping movements; even with forces of lower intensity tend to induce extensive areas of hyalinization.

\section{Probing depth}

The present study showed that there was no statistically significant difference between the two groups at all intervals. But it showed significant difference in each group when periods of treatment compared to base time. The reason might be due to stimulation of plaque retention at the bracket which causes gingival inflammation ${ }^{(21)}$.

\section{Gingival and plaque index}

The current study showed insignificant difference in all periods when compared with base time in all groups. The subjects in the present study showed, however, similar gingival conditions for the test and control teeth. These findings were in accordance with a previous study ${ }^{(22)}$ that monitored no significant changes were observed on any of the clinical parameters during the whole experimental period. This is mainly due to the oral hygiene instructions given to each patient before and during the experimental treatment. In fact, meticulous plaque control and motivation for oral hygiene took place every week for all patients. These finding in disagreement with a previous study ${ }^{(23)}$ reported that, even after maintaining seemingly excellent oral hygiene, patients usually experience mild to moderate gingivitis within 1-2 months of fixed appliance placement with bands. These differences were attributed mainly to the mechanical irritation by the orthodontic bands which are more likely to come in contact with the gingiva around the posterior rather than the anterior teeth.

\section{CONCLUSIONS}

Within the limitations and findings of the present study it could be concluded that:

1. Level of lactate dehydrogenase enzyme showed significant elevation at two week after treatment in both two groups followed by slight decrease to four week after treatment.

2. The level of lactate dehydrogenase enzyme in gingival crevicular fluid could be used as a biomarker for periodontal metabolism related to orthodontic tooth movement.

3. The level of lactate dehydrogenase enzyme did not show significant difference among different tooth malpositions during correction by orthodontic treatment.

4. Generally there was no correlation between lactate dehydrogenase enzyme level and all measured clinical parameters (gingival index, plaque index and probing depth) during correction of different tooth malpositions.

\section{REFERENCES}

1. Krishnan V and Davidovitch Z. Cellular, molecular, and tissue-level reactions to orthodontic force. Am J Orthod Dentofacial Orthop. 2006; 129:1-32.

2. Alfaqeeh $\mathrm{S}$ and Anil S.Gingival Crevicular Fluid Flow Rate and Alkaline Phosphatase Level as Potential Marker of Active Tooth Movement. Oral Health Dent Manag J.2014; 13:458-63.

3. Garlet T, Coelho U, Repeke C, Silva J, Cunha F, Garlet $\mathrm{G}$.Differential expression of osteoblast and osteoclast chemo attractants in compression and tension sides during orthodontic movement. J Cyto. 2008; 42: 330-5.

4. Davidovitch Z. Tooth movement. Crit Rev Oral Biol Med. 1991;2:411-50.

5. Krishnan V and Davidovitch Z. On a path to unfolding the biological mechanisms of orthodontic tooth movement. J Dent Res. 2009; 88:597-608.

6. Kitase Y, Yokozeki M, Fujihara S, Izawa T, Kuroda S, Tanimoto K, Moriyama K , Tanaka E. Analysis of gene expression profiles in human periodontal ligament cells under hypoxia: the protective effect of $\mathrm{CC}$ chemokine 
ligand 2 to oxygen shortage. Arch Oral Biol. 2009 ;45: 618-24.

7. Yamamoto Z, Jaafar I, Senafi S. Orthodontic treatment effects on inflammatory marker profiles in saliva before and after 2 archwire changes. AIP Conf. Proc. 2014 ;1571:208-14.

8. Davidovitch Z and Krishnan V. Biological mechanism of tooth movement . 2 nd edition . Willey Blackwell . 2015 ; ch 5:62-81.

9. King G, Keeling S , Wronski J. Histomorphometric study of alveolar bone turnover in orthodontic tooth movement. Bone Res.1991;12: 401-9.

10. Von Böhl M, Maltha J, Von Den Hoff J, Jagtman AM. Focal hyalinization during experimental tooth movementin beagle dogs. Am J Orthod Dentofacial Orthop. 2004 ;125:615-23.

11. Hisham S, Ariffin Z, Yamamoto Z, Abidin I. Cellular and molecular changes in orthodontic tooth movement. World J Orthod . 2011; 11:1788-803.

12. Domenico M, D'apuzzo F , Feola A, Cito L, Monsurrò A, Pierantoni $\mathrm{G}$, et al. Cytokines andVEGF induction in animal models. J Biomed and Biotech. 2012; ID 201689: 4.

13. Dannan A, Darwish M, Sawan M. Effect of Orthodontic Tooth Movement on Gingival Crevicular Fluid Infiltration; a Preliminary Investigation. J of Dent. 2009 ;6:109-15.

14. Perinetti G, Paolantonio M, D'Attilio M, D'Archivio D, Dolci M, Femminella B, Festa F , Spoto G. Aspartate aminotransferase activity in gingival crevicular fluid during orthodontic treatment. A controlled short-term longitudinal study. J Periodontol. 2003 ;74:145-52.
15. Coquelle N, Fioravanti E, Weik M, Vellieux F, Madern D. Activity, Stability and Structural Studies of Lactate Dehydrogenases Adapted toExtreme Thermal Environments. J Mol Biol. 2007 ;374:547-62

16. Ariffin S, AbuKasim A, AbdulWahab R. Lactate Dehydrogenase Activity during Tooth Movement under $1.0 \mathrm{~N}$ and $1.5 \mathrm{~N}$ Continuous Force Applications. Sains Malaysiana. 2013 ;42: 99-105.

17. Mannen H, Tsoi S, Krushkal J. The DNA cloning and molecular evolution of reptile and pigeon lactate dehydrogenase isozymes.Mol Biol Evol.1997 ;14:1081-7.

18. Ramussen L, Hansto L, Lerner H. Characterization of bone resorbing activity in gingival crevicular fluid from patients with periodontitis. J Clin Periododontol. 2000;27:41-52.

19. Burstone C. The biomechanics of tooth movement. Philadelphia: Lea \& Febiger.1962 :197-213.

20. Consolaro A. Force distribution is more important than its intensity. Dent Press J Orthod. 2014 ;19:5-7.

21. Paolantonio M, diGirolamo G, Pedrazzoli, V, di Murro C , Picciani, C. Occurrence of Actinobacillus actinomycetemcomitans in patients wearing orthodontic appliances. A cross-sectional study. J Clin Periodontol.1996; 23:112-8.

22. Giannopoulou C, Dudic A, Montet X, Kiliaridis S. Periodontal parameters and cervical root resorption during orthodontic tooth movement. Mombelli J Clin Periodontol. 2008; 35:501-6.

23. Zachrisson $S$ and Zachrisson B.Gingival condition associated with orthodontic treatment. Angle Orthod. 1972; 42:26-34. 